\title{
International registry of congenital porto-systemic shunts A multi-centre, retrospective and prospective registry of neonates, children and adults with congenital porto-systemic shunts
}

\section{Simona KORFF ( $\square$ simona.korff@hcuge.ch )}

University Hospital Geneva: Hopitaux Universitaires Geneve https://orcid.org/0000-0001-9049-2210

Khaled Mostaguir

Geneva University Hospitals: Hopitaux Universitaires Geneve

Maurice Beghetti

Geneva University Hospitals: Hopitaux Universitaires Geneve

Lorenzo D'Antiga

Papa Giovanni XXIII Hospital: Aziende Socio Sanitarie Territoriale Papa Giovanni XXIII

Dominique Debray

Assistance Publique Hopitaux de Paris: Assistance Publique - Hopitaux de Paris

\section{Stéphanie Franchi-Abella}

Assistance Publique - Hôpitaux de Paris: Assistance Publique - Hopitaux de Paris

\section{Emmanuel Gonzales}

Assistance Publique - Hôpitaux de Paris: Assistance Publique - Hopitaux de Paris

Florent Guerin

Assistance Publique - Hôpitaux de Paris: Assistance Publique - Hopitaux de Paris

Anne-Lise Hachulla

Geneva University Hospitals: Hopitaux Universitaires Geneve

Virginie Lambert

Assistance Publique Hopitaux de Paris: Assistance Publique - Hopitaux de Paris

\section{Periklis Makrythanasis}

National and Kapodistrian University of Athens: Ethniko kai Kapodistriako Panepistemio Athenon

Nicolas Roduit

Geneva University Hospitals: Hopitaux Universitaires Geneve

\section{Laurent Savale}

Assistance Publique Hopitaux de Paris: Assistance Publique - Hopitaux de Paris

\section{Marie-Victoire Senat}

Assistance Publique Hopitaux de Paris: Assistance Publique - Hopitaux de Paris

Joël Spaltenstein

Geneva University Hospitals: Hopitaux Universitaires Geneve 


\section{Frank van Steenbeek}

Utrecht University: Universiteit Utrecht

\section{Barbara E. Wildhaber}

Geneva University Hospitals: Hopitaux Universitaires Geneve

\section{Marcel Zwahlen}

University of Bern: Universitat Bern

Valérie A. McLin

Geneva University Hospitals: Hopitaux Universitaires Geneve

\section{Research Article}

Keywords: Congenital, portosystemic, shunt, registry, liver, rare disease.

Posted Date: February 18th, 2022

DOI: https://doi.org/10.21203/rs.3.rs-1294835/v1

License: (c) (1) This work is licensed under a Creative Commons Attribution 4.0 International License.

Read Full License 


\title{
International registry of congenital porto-systemic shunts
}

\author{
A multi-centre, retrospective and prospective registry of neonates, children
}

\author{
and adults with congenital porto-systemic shunts
}

Simona Korff ${ }^{1}$, Khaled Mostaguir ${ }^{2}$, Maurice Beghetti ${ }^{3}$, Lorenzo D'Antiga ${ }^{4,5}$,

Dominique Debray ${ }^{5,6}$, Stéphanie Franchi-Abella ${ }^{5,7}$, Emmanuel Gonzales $^{5,8}$,

Florent Guerin ${ }^{5,9}$, Anne-Lise Hachulla $^{10}$, Virginie Lambert ${ }^{11}$, Periklis

Makrythanasis ${ }^{12}$, Nicolas Roduit ${ }^{13}$, Laurent Savale ${ }^{14}$, Marie-Victoire Senat ${ }^{15}$, Joël

Spaltenstein ${ }^{16}$, Frank van Steenbeek ${ }^{17}$, Barbara E. Wildhaber ${ }^{1}$, Marcel

Zwahlen ${ }^{18}$, Valérie A. McLin ${ }^{1,5}$

${ }^{1}$ Swiss Pediatric Liver Center, University Hospitals Geneva, Department of Pediatrics, Gynecology, and Obstetrics, University of Geneva, Geneva, Switzerland

${ }^{2}$ Clinical Research Centre, Geneva University Hospitals and Faculty of Medicine, University of Geneva, Geneva, Switzerland

${ }^{3}$ Congenital Heart Center, Division of Pediatric Subspecialities, University Hospitals Geneva,

Department of Pediatrics, Gynecology, and Obstetrics, University of Geneva, Geneva,

Switzerland

${ }^{4}$ Paediatric Hepatology, Gastroenterology and Transplantation, Hospital Papa Giovanni XXIII, Bergamo, Italy

${ }^{5}$ ERN RARE LIVER

${ }^{6}$ Assistance Publique Hôpitaux de Paris (AP-HP), Université de Paris, Competence center for rare vascular diseases, Necker Hospital, Pediatric liver unit, Paris, France

${ }^{7}$ Assitance Publique Hôpitaux de Paris (AP-HP), Paris-Saclay University, Bicêtre Hospital, Pediatric radiology department, Le Kremlin-Bicêtre, France Paris, France

${ }^{8}$ Assitance Publique Hôpitaux de Paris (AP-HP), Paris-Saclay University, Pediatric, Hepatology and Liver Transplantation, Reference center for liver vascular diseases, FSMR FILFOIE, Hépatinov, Inserm U 1193, Bicêtre Hospital, Le Kremlin-Bicêtre, France

${ }^{9}$ Assitance Publique Hôpitaux de Paris (AP-HP), Paris-Saclay University, Bicêtre Hospital,

Department of Paediatric Surgery, Le Kremlin-Bicêtre, France

${ }^{10}$ Division of radiology, University Hospitals Geneva, Geneva, Switzerland

${ }^{11}$ Assitance Publique Hôpitaux de Paris (AP-HP), Paris-Saclay University, Hospital,

Department of Paediatric Radiology, Le Kremlin-Bicêtre ; Congenital Cardiology Montsouris, Institut Mutualiste Montsouris, Paris, France

${ }^{12}$ Laboratory of Medical Genetics, Medical School, National and Kapodistrian University of Athens, Athens, Greece; Department of Genetic Medicine and Development, Medical

School, University of Geneva, Geneva, Switzerland; Biomedical Research Foundation of the Academy of Athens, Athens, Greece

${ }^{13}$ Information systems department, University Hospitals Geneva, Geneva, Switzerland

${ }^{14}$ Paris-Saclay University, Faculty of Medecine, Le Kremlin-Bicêtre, France; Assitance

Publique Hôpitaux de Paris (AP-HP), French national reference center for pulmonary

hypertension, Department of Pulmonology and Respiratory Intensive Care, Bicêtre Hospital, 
Le Kremlin-Bicêtre, France; INSERM UMR_S 999, Marie Lannelongue Hospital, Le Plessis-

Robinson, France

${ }^{15}$ Assitance Publique Hôpitaux de Paris (AP-HP), Paris-Saclay University, Bicêtre Hospital, Gynecology and obstetrics department, Paris, France

${ }^{16}$ OsiriX Foundation, Geneva, Switzerland

${ }^{17}$ Utrecht University, Faculty of Veterinary Medicine, Department of Clinical Sciences, Utrecht, The Netherland

18 University of Bern, Institute of Social and Preventive Medicine, Bern, Switzerland / http://orcid.org/0000-0002-6772-6346

\section{Corresponding author:}

Simona Korff PhD

Swiss Pediatric Liver Center

6 rue Willy Donzé

1205 Geneva

Switzerland

Email: simona.korff@hcuge.ch

Phone +41223724545

\section{Abstract: \\ Background:}

Congenital portosystemic shunts (CPSS) are rare vascular malformations associated with the risk of life-threatening systemic conditions, which remain underdiagnosed and often are identified after considerable diagnostic delay. CPSS are characterized by multiple signs and symptoms, often masquerading as other conditions, progressing over time if the shunt remains patent. Which patients will benefit from shunt closure remains to be clarified, as does the timing and method of closure. In addition, the etiology and pathophysiology of CPSS are both unknowns. This rare disorder needs the strength of numbers to answer these questions, which is the purpose of the international registry of CPSS (IRCPSS).

\section{Method:}

A retrospective and prospective registry was designed using secuTrial ${ }^{\circledR}$ by the ISO certified Clinical Research Unit. Given that a significant number of cases entered in the registry are retrospective, participants have the opportunity to use a semi-structured minimal or complete data set to facilitate data entry. In addition, the design allows subjects to be entered into the IRCPSS according to clinically relevant events. Emphasis is on longitudinal follow-up of signs and symptoms, which is paramount to garner clinically relevant information to eventually orient patient management. The IRCPSS includes also three specific forms to capture essential radiological, surgical, and cardiopulmonary data as many times as relevant, which are completed by the specialists themselves. Finally, connecting the clinical data registry with a safe image repository, using state-of-the-art pseudonymization software, was another major focus of development. Data quality and stewardship is ensured by a steering committee. All centers participating in the IRCPSS have signed a memorandum of understanding and obtained their own ethical approval.

\section{Conclusion:}

Through state-of-the-art management of data and imaging, we have developed a practical, user-friendly, international registry to study CPSS in neonates, children, and adults. Via this 
multicenter and international effort, we will be ready to answer meaningful and urgent questions regarding the management of patients with CPSS, a condition often ridden with significant diagnostic delay contributing to a severe clinical course. We look forward to working with an ever expanding network of collaborators.

\section{Keywords}

Congenital, portosystemic, shunt, registry, liver, rare disease.

\section{Background}

Congenital portosystemic shunts (CPSS) are a rare malformation in humans accepted to result from incomplete vascular remodeling during embryogenesis, whereby the blood leaving the intestine does not pass entirely through the liver. CPSS are broadly divided into two categories: intrahepatic $(\mathrm{IH})$ and extrahepatic $(\mathrm{EH})$. The significance of this distinction is twofold. First, they are anatomically different and therefore the relative proportion of portosystemic bypass differs between forms, with EH shunts often associated with a much more significant amount of splanchnic blood derivation than IH. Second, IH seem to be more likely to close spontaneously early in life (18).

The systemic repercussions of abnormal portosystemic shunting and decreased hepatic portal flow are numerous and potentially serious. They include signs and symptoms such as hepatic encephalopathy, pulmonary vascular diseases (hepatopulmonary syndrome or pulmonary arterial hypertension), benign or malignant liver tumors, and biological and metabolic disorders $(1,4,5,11)$. Other less well-described associations include in utero growth retardation or tall stature in infancy, nephropathy, thyroid dysfunction, and coagulation abnormalities. In adults, CPSS have been reported at all ages, and are often reported as incidental findings, although in referral centers they are now sought as part of the work up of pulmonary hypertension (2).

The true incidence of CPSS is unknown, but it is estimated to affect $1 / 30-50$ '000 live births $(3,4)$. CPSS is also known to be associated with other malformations or syndromes, such as congenital heart disease, heterotaxy, polysplenia syndrome, chromosomal anomalies, cutaneous or hepatic hemangiomas 1,4,12). CPSS can be diagnosed at any age, either because the patient is symptomatic or as an incidental finding. Given their rarity, patients presenting with symptoms of systemic complications may experience significant diagnostic delay, because many of CPSS signs and symptoms mimic other common paediatric conditions. Spontaneous closure of the CPSS has been reported to occur in some anatomic forms during the first one to two years of life (18). When the CPSS remains patent, radiologic or surgical closure of the CPSS may prevent, resolve, or stabilize complications $(5,11,12,13)$. Yet, the conspicuous gap to fill in our current knowledge is which patient will benefit from shunt closure and when.

Therefore, the main aim of the International Registry of Congenital PortoSystemic Shunts (IRCPSS) is to characterize the clinical course of patients with CPSS and to identify subjects at risk of developing systemic complications with the overarching goal to stratify risks and standardize care of patients with CPSS. Although CPSS are increasingly suspected and sought in specialized centers, much is still unknown clinically, histologically, and biologically. 
Secondary aims include i) developing a clear, consensual nomenclature; ii) correlating prenatal findings with post-natal anatomy; and iii) unravelling the developmental mechanisms of CPSS.

\section{Methods}

\section{Study design}

Given the rarity of CPSS, it is crucial to emphasize the importance of recording both prevalent and incident cases and to include as many centers as possible. It is for these reasons that the registry is designed as both a retrospective and prospective cohort study. At the time of print, it is estimated that $>500$ retrospective cases are currently followed by centers participating in the IRCPSS. Although medical records may be incomplete, we aim to include all cases in the registry given the rarity of the condition and the importance of garnering sufficient numbers to improve the understanding of the full spectrum of these rare malformations.

\section{- Descriptive Data}

The following parameters are essential to improve our understanding of the medical course of patients with CPSS: medical history, anatomical characteristics of the CPSS, radiological progression of the CPSS over time, and the longitudinal follow up of signs and symptoms over time. Therefore the registry is designed to include clinical, imaging, histological, and biological data collected from the day of diagnosis until five years after CPSS closure (spontaneous or procedural), or any clinically relevant visit thereafter. As this is not an interventional trial, each participating center sets its own clinical follow-up schedule and enters into the registry what the site principal investigator (PI) deems to be clinically relevant visits (Figure 1).

Title Figure 1 - Longitudinal follow-up of patients with CPSS

\section{- Imaging}

Imaging is crucial to diagnose and characterize CPSS, and to develop a clear and consensual nomenclature. Images are collected in all collaborating centers and stored in a dedicated imaging server meeting all current security norms. Scanned histology slides can be uploaded to the same server. Images are linked to the clinical registry using a secure process described below (image management).

\section{Study population}

Since the registry aims to understand the full spectrum of CPSS, the IRCPSS includes neonates, children, and adults and follows subjects longitudinally. Any neonate, child or adult with CPSS is eligible for inclusion. Patients diagnosed in utero are enrolled at birth. Patients with chronic liver disease and secondary shunts are excluded.

\section{Model conception}

Understanding the clinical question is central to developing the optimal registry. In the case of CPSS the crux of managing patients is to determine who will develop systemic complications, who will close spontaneously, and consequently who will need shunt closure, and when. A preliminary analysis of the first cases enrolled in the registry suggested that $\mathrm{EH}$ shunts (versus $\mathrm{IH}$ ) are unlikely to close spontaneously, and have been shown to be associated with at least one severe complication in almost $70 \%$ of cases (14), confirming earlier reports (1). Still, while this preliminary analysis helps guide which patients warrant a closer follow-up, it is not determined yet which patients may not need surgical or radiological closure, both 
procedures being laden with risks of significant complications, something which the registry aims to capture. Furthermore, it remains to be determined, among those patients who will develop associated signs or symptoms, which of these will regress following shunt closure, and which will need further specialized treatment. One such example is the management of liver nodules following shunt closure: while some may regress, others may progress to hepatocellular carcinoma. This question holds true for the other systemic signs and symptoms associated with CPSS: pulmonary vascular disease, cognitive disability, endocrine anomalies. Therefore, the IRCPSS needs to capture longitudinal information in order to offer investigators the opportunity to analyze these very specific questions in nested studies.

There are several other challenging aspects of managing patients with CPSS which the registry aims to tackle. Among them, some of the primordial questions include how to proceed with complex shunts, how to follow up shunts diagnosed in utero, and the complexity of managing CPSS in the setting of congenital heart disease. Concerning complex shunts, a significant fraction of patients will present with more than one portosystemic communication, possibly requiring multiple procedures for closure. This particular subset of patients warrants careful attention in order to determine if complete shunt closure is mandatory or if residual, but minimal portosystemic bypass is clinically tolerable. In the registry each complex shunt is identified and has its own classification to facilitate follow-up through multiple closures. If a new shunt is identified, it can be managed independently from the first one. Concerning shunts diagnosed in utero, our preliminary study (14) suggested that a large fraction of these resolves spontaneously. However, the registry will contribute identifying which patients warrant ongoing follow-up would help individualize care. The main clinical questions and goals of the IRCPSS determined the parameters (Table 1 ) as well as the structure of the registry (Table 2).

Table 1 - List of parameters recorded in the IRCPSS

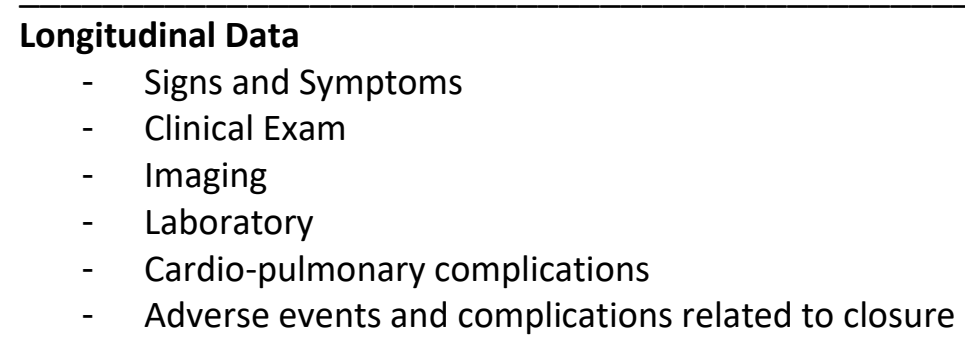

\section{Crossectional Data}

- Inclusion criteria

- Demographics

- Medical history

- Pre-natal history

- Mode of presentation

- Associated malformations (pre- and post-natal)

- Shunt classification (pre- and post-natal)

- Indication for closure

- Type of closure and procedure

- Available tissue samples and genetic workup

- Termination of follow up 
Table $\mathbf{2}$ - Parameters recorded in the IRCPSS related to the main clinical questions and goals

\begin{tabular}{lll}
\hline Clinical questions and goals & Crossectional Data & Longitudinal Data \\
\hline $\begin{array}{l}\text { Clear and consensual } \\
\text { nomenclature }\end{array}$ & Shunt classification linked to images & \\
\hline $\begin{array}{l}\text { Correlation of prenatal } \\
\text { findings with post-natal } \\
\text { anatomy }\end{array}$ & $\begin{array}{l}\text { Patient medical history, mode of } \\
\text { presentation, associated } \\
\text { malformations, shunt classification }\end{array}$ & \\
\hline $\begin{array}{l}\text { Type of cardiopulmonary } \\
\text { complication and prognosis }\end{array}$ & Mode of presentation & $\begin{array}{l}\text { Cardio-pulmonary } \\
\text { complications }\end{array}$ \\
\hline $\begin{array}{l}\text { Who will develop associated } \\
\text { signs or symptoms? }\end{array}$ & $\begin{array}{l}\text { Pre-natal history, pas medical } \\
\text { history, associated malformations, } \\
\text { mode of presentation, shunt } \\
\text { classification }\end{array}$ & $\begin{array}{l}\text { Signs and symptoms before } \\
\text { closure }\end{array}$ \\
\hline $\begin{array}{l}\text { Which signs or symptoms } \\
\text { regress after shunt closure } \\
\text { and which will need further } \\
\text { specialized treatment }\end{array}$ & $\begin{array}{l}\text { Associated malformations, shunt } \\
\text { classification, mode of presentation, } \\
\text { indication for closure, type of closure } \\
\text { and procedure }\end{array}$ & $\begin{array}{l}\text { Signs and symptoms, clinical } \\
\text { exams, imaging, laboratory, } \\
\text { adverse events and } \\
\text { complications }\end{array}$ \\
\hline $\begin{array}{l}\text { Closure, who and when? } \\
\text { closure? }\end{array}$ & $\begin{array}{l}\text { Associated malformations, shunt } \\
\text { classification, mode of presentation, } \\
\text { indication for closure, type of closure } \\
\text { and procedure }\end{array}$ & $\begin{array}{l}\text { Shunt identification, signs and } \\
\text { symptoms, clinical exam, } \\
\text { imaging, laboratory, adverse } \\
\text { events and complications }\end{array}$ \\
\hline $\begin{array}{l}\text { How to proceed with } \\
\text { complex shunts? }\end{array}$ & $\begin{array}{l}\text { Signs and symptoms before and } \\
\text { after closure }\end{array}$ \\
\hline & & \\
\hline & &
\end{tabular}

\section{Clinical data collection}

Only available data collected as part of routine clinical care of each center are recorded in the registry; additional medical visits or lab draws are not necessary for participation. Consents are given by the adult subjects or by the parents or legal representatives of children subjects following the ethical legislation of the country.

There are two semi-structured data sets to enter data. A Minimal Data Set (MDS) is available to include and to facilitate data entry for historical cases whose files are often very incomplete. The MDS does not include pre-natal data, medical history, pre-closure visits, or surgical or radiological information at closure. In turn, the Complete Data Set (CDS) is designed for prospective data and complete retrospective data. It includes pre-natal data and data listed in Table 1.

Data collected at each visit include demographic data (age, sex, weight, and height), clinical exams, and laboratories. Information on the type of imaging performed is also captured (angiography, magnetic resonance imaging, scintigraphy, ultrasound, computed tomography, electrocardiogram). Signs and symptoms are tracked at each visit in order to follow their 
course longitudinally before and after shunt closure (Figure 2). For quantifiable data, such as liver nodules, it is possible to follow number and size over time.

Title Figure 2 - Data concepts, structure, and longitudinal collection of the complete data set Legend Figure 2 - UML presentation: package/class \& use case diagram - relationships: $0 . .1$ no instance or 1 instance, $0 .{ }^{*}$ zero or more instance, $1 . .^{*}$ at least one instance Purple: visits over time Yellow: clinical forms and imaging Green: specific forms at the Basic Data \& Diagnosis visit Red: specific forms at closure Grey: Specific forms at any time-point

Additionally, some data are collected at specific time-points during follow-up. At the first visit - also called "Basic Data \& Early Diagnosis visit" - the following are recorded: patient medical history, shunt mode of presentation, and anatomical classification according to imaging (19). Associated malformations and prenatal data are also recorded by the system when available. Specific surgical and radiological data are collected during the closure visit. Cardio-pulmonary complications are documented and followed in specific and independent cardio-pulmonary forms and can be collected/recorded at any time-point. There are three (f3) specific forms meaning they are meant to be entered independently by specialists: cardiopulmonary, surgery, interventional radiology (Figure 2). Given that cardiopulmonary clinically relevant visits be required at different time points than shunt-specific visits, these can be entered independently.

If the evolution of the CPSS goes well, the normal termination is at five years after the last closure or at the last visit if the follow-up of the subject is shorter.

\section{Data management and collection}

Data management is performed by the Clinical Research Center at Geneva University Hospitals and Faculty of Medicine. The unit is certified ISO 9001/2008, and guarantees full compliance with Good Clinical Practices (GCP).

Physical and electronic access to the data center is logged and limited to authorized personnel. Vulnerability testing is performed regularly to reduce potential exposure. The internal network is protected by multiple firewalls, proxy, reverse-proxy and state-of-the-art anti-virus solutions.

Data are managed and physically stored using secuTrial ${ }^{\circledR}(15)$, a professional, specialized, browser-based, and GCP-compliant system for collecting, validating, and visualizing patient data in clinical research trials and registries. secuTrial ${ }^{\circledR}$ was adapted specifically to the complex needs of the registry.

Data collected for each research subject constitute the subject's electronic Case Report Form (eCRF). Each eCRF includes the parameters and follows the structure previously described under "Clinical Data Collection".

Research subjects are automatically pseudonymized and access to their data is strictly limited to authorized users. No personal data or data that may easily identify subjects are recorded, also for imaging. Each participating center has its own dedicated and protected area. The forms containing the data are edited and accessed by the study personnel based on a predefined set of roles and authorizations. 
All system logins, interventions, data modifications, and form status changes are thoroughly recorded for audit purposes.

\section{Data quality}

The implementation of the data model was designed to avoid redundancy, to facilitate data capture, while minimizing logic and typing errors (e.g. automatic conversion of laboratory values, assessment of newly entered values with regard to previously entered ones, etc.).

In addition to the automatic quality rules, data validation and review is thoroughly ensured by members of the registry steering committee (SC). To facilitate data and activity monitoring, up-to-date graphical and text reports, as well as descriptive statistics, are available in real time (Figure 3a). Data management is further strengthened by an interactive, electronic process to comment, query and claim corrections, thereby ensuring high data quality.

\section{Navigability}

The built-in graphical interface has been optimized to facilitate navigability as much as possible during the data capture and data validation processes (Figure 3b). Color codes indicate completion, validation, and accuracy status of the different data sections facilitating navigation between different visits and clinical categories (or sub-domains) by investigators.

Title Figure 3a - secuTrial ${ }^{\circledR}$ : overview of the data entry progress and completion that each available in real time for each center

Title Figure $3 \mathbf{b}$ - secuTrial ${ }^{\circledR}$ : overview of subject visit plan and forms

\section{Image management}

In addition to clinical data, the project includes DICOM imaging data that has been deidentified using the KARNAK (16) gateway. All personally identifying information (PII) is removed from non-pixeldata DICOM attributes. In cases such as ultrasound imaging, where PII is often burnt-in to the pixeldata, the images are modified to remove the identifying information.

Image management is performed within a dedicated image server built on the KHEOPS platform (17). The KHEOPS platform provides specific access controls based on users' needs and an organizational structure, such as images submitted by different recruitment centers, remain isolated from each other.

The image server is hosted in the data center of the Campus Biotech in Geneva under the responsibility of the Institute of Translational Molecular Imaging. This institute is affiliated with the University of Geneva and the Swiss Federal Institute of Technology.

Within secuTrial ${ }^{\circledR}$, each patient eCRF includes a redirection link that ensures a dynamic connection between the clinical registry and the image server.

\section{Data analysis}

The planned statistical analyses are partly of a descriptive and partly of a comparative nature. Examples of descriptive analyses include describing the prevalence and incidence of a particular extrahepatic complication related to CPSS: for example pulmonary hypertension, 
liver nodules or tall stature. Each sign or symptom can in turn be studied by describing associations with neonatal findings or analyzing severity, treatment, and outcomes.

Comparative analyses will compare demographic or clinical characteristics between patients with or without a given sign or symptom: for example looking for differences between patients who develop pulmonary hypertension and those who do not. Among those who do, functional characteristics (for example New York Heart Association functional class) will be compared and regression models developed with the aim to identify associations.

\section{Description of recruiting centers}

The first step for a new center to be part of the IRCPSS is to obtain the approval of its ethics committee. To facilitate the procedure we propose consent forms in English, French, German, and Italian. If needed a Data Transfer Agreement (DTA) model has also been established by the legal department of University Hospitals Geneva to govern the transfer, use, and protection of data.

CPSS is a complex disease that requires multidisciplinary management. Therefore, it is expected that each collaborating center include a hepatologist, surgeon, radiologist, cardiologist, and pulmonologist on their CPSS team. To this end, the IRCPSS requires the participation of these specialists to enroll patients in the registry, given the highly specialized nature of the data, beyond the expertise of most research coordinators. The site PI of each center oversees the coordination between all these specialties and each center decides who enters what data in the registry, depending on the configuration and availability of resources. However, it is expected that cardiologists enter their data, given the complexity of the specific reports. For data quality issues, each specialist validates the data for his particular subspecialty in his center, and a second level of validation is performed by the SC.

The prototypic participating center is a recognized national and international center for liver disease with expertise in CPSS diagnosis and management in children or adults. At the time of print, 50 centers in 22 countries are part of the registry, 17 of which have obtained the approval of their ethics committee and 11 have initiated data entry.

\section{Data stewardship and publications}

All centers participating in the IRCPSS have access to their own data and may agree to share their data with another center under the auspices of an ancillary project validated by the SC. Members of the SC have access to all data for oversight and quality control. Publication policy is defined by the SC in a Memorandum of Understanding signed by all centers entering data in the registry. Authorship is determined according to the scientific contribution of an individual to a given project or manuscript, and patient enrollment is acknowledged by listing all site contributors in alphabetical order in the 'contributors' section as found in PubMed. Partners wishing to conduct a nested study may submit to the SC a short study proposal for review and approval by the SC (contact via www.ircpss.com).

\section{Discussion and conclusion}

The purpose of the IRCPSS registry is to bring together a vast collection of longitudinal, clinical, and imaging data across several systems in order to deeply characterize the clinical course of patients with CPSS to allow for harmonizing clinical decision making and improving patient 
management. The challenge has been to build a solid and suitable data model encompassing the various clinical sub-domains and their complexity, while remaining as flexible as possible to facilitate data capture, ease of use, and long-term adaptability. We believe that we have successfully designed a tool that also offers the required flexibility to capture data across the many participating centers. It also offers the possibility for future technical evolution, including towards the development of interventional studies.

CPSS are a rare condition, and previous single-center studies have not been powered enough to answer some of the key questions that this large multicenter registry will be able to tackle. One of the unique features of this registry is that it brings together longitudinal data about the CPSS itself, with that of several organ systems in order to uniquely follow the course of systemic signs and symptoms over time. Furthermore, the creation of the IRCPSS has garnered the interest of multiple centers across continents. Importantly, it has succeeded in securing the commitment of multidisciplinary teams in all these centers. This is essential for developing a detailed understanding of CPSS and stems from the existent reality of clinical practice in the respective centers. In most instances, patients with CPSS cannot be managed in isolation, but rather need the collective know-how of multidisciplinary teams comprised of interventional radiologists, hepatologists, surgeons, endocrine specialists, pulmonologists and cardiologists, an aspect which this registry also aims to favor.

The multi-center approach of the IRCPSS gave rise to a series of conferences where all the collaborating centers have the opportunity to present and discuss their complex clinical cases on the multidisciplinary expertise of the SC and experts of other centers. A way for the members of the SC to share their expertise and experience, as well as standardize care of patients with CPSS.

Finally, CPSS are complex vascular malformations with potentially severe systemic consequences often masquerading as other common complaints. Increasing awareness is therefore paramount and the creation of the IRCPSS enhance the visibility of this rare vascular malformation.

\section{List of abbreviations}

CPSS - Congenital portosystemic shunts

IRCPSS - International Registry of Congenital PortoSystemic Shunts

IH - intrahepatic

EH - extrahepatic

MDS - Minimal Data Set

CDS - Complete Data Set

GCP - Good Clinical Practices

eCRF - electronic Case Report Form

$\mathrm{SC}$ - steering committee

ITMI - Institute of Translational Molecular Imaging

EPFL - Swiss Federal Institute of Technology

DTA - Data Transfer Agreement

$\mathrm{PI}$ - Principal Investigator

\section{Ethics approval and consent to participate}


Ethical approval of the sponsor was granted by the Geneva cantonal Research Ethics Commission (CCER) in Switzerland on the 26 $6^{\text {th }}$ of April 2018 (2018-00413). All other centers participating in the IRCPSS have obtained their own ethical approval and all subjects gave informed consent.

\section{Consent for publication}

Not applicable.

\section{Availability of data}

Not applicable.

\section{Competing interests}

Lorenzo D'Antiga serves on the advisory board or and/or consults for the following companies: Selecta, Vivet, Spark, Alexion, Mirum, Albireo. The other authors have not competing interests related to the content presented in this manuscript.

\section{Funding}

The IRCPSS is funded by a network grant from the European Society for Pediatric Gastroenterology, Hepatology and Nutrition and by a Registry Grant from the European Association for the Study of the Liver. It has garnered support from the Ferrari Foundation and the Prim'Enfance Fondation in Geneva, Switzerland, and benefits from operational support from the University Hospitals Geneva, and The University of Geneva. Collaborators include the European Reference Network RARE-LIVER, the Vascular Liver Disease Group (VALDIG), the Centre de Référence Maladies Vasculaires du Foie, and the Filière de Santé Maladies Rares du Foie de l'Adulte et de l'Enfant (FILFOIE)

\section{Authors' contributions}

SK, KM, MB, DD, SFA, EG, FG, VL, LS, BW and VM conceptualized and designed the registry. They are actively involved in the implementation of the registry at their institutions. SK, KM and VM wrote the manuscript. All authors read and approved the final manuscript.

\section{Acknowledgements}

The authors thank Lilianna Bolliger of the Institute of Social and Preventive Medicine (ISPM) for her guidance during the registry conception, Antoine Poncet of the clinical epidemiology service of the University Hospitals Geneva for his statistical advice, the numerous clinicians who contributed to eCRF conception, and to the numerous families who have consented to contribute to bettering our understanding of CPSS.

\section{REFERENCES}

1. Bernard O, Franchi-Abella S, Branchereau S, et al. Congenital portosystemic shunts in children: recognition, evaluation, and management. Semin Liver Dis 2012;32(4):27387. 
2. Krowka MJ, Fallon MB, Kawut SM, et al. International Liver Transplant Society Practice Guidelines: Diagnosis and Management of Hepatopulmonary Syndrome and Portopulmonary Hypertension. Transplantation 2016;100(7):1440-52.

3. Sakura N, Mizoguchi N, Eguchi T, Ono H, Mawatari H, NaitouK, et al. Elevated plasma bile acids in hypergalactosaemicneonates: a diagnostic clue to portosystemic shunts. Eur J Pedi-atr 1997;156:716-8.

4. Sokollik C, Bandsma RH, Gana JC, van den Heuvel M, Ling SC. Congenital portosystemic shunt: characterization of a multi-system disease. J Pediatr Gastroenterol Nutr 2013;56:675-81.

5. McLin VA, Franchi Abella S, Debray D, Guérin F, Beghetti M, Savale L, et al. Congenital Portosystemic Shunts: Current Diagnosis and Management. J Pediatr Gastroenterol Nutr 2019; 68:615-22.

6. Van Steenbeek FG, et al., Inherited liver shunts in dogs elucidate pathways regulating embryonic development and clinical disorders of the portal vein. Mamm Genome, 2012; 23(1-2):76-84.

7. Berent AC, Tobias KM. Portosystemic vascular anomalies. Vet Clin North Am Small Anim Pract, 2009; 39(3):513-41.

8. Martin RA. Congenital portosystemic shunts in the dog and cat. Vet Clin North Am Small Anim Pract. 1993;23(3):609-23.

9. Cudalbu, $\mathrm{C}$, et al. The $\mathrm{C} 57 \mathrm{BL} / 6 \mathrm{~J}$ mouse exhibits sporadic congenital portosystemic shunts. PLoS One. 2013; 8(7):e69782.

10. Lahvis GP, et al. Portosystemic shunting and persistent fetal vascular structures in aryl hydrocarbon receptor-deficient mice. Proc Natl Acad Sci U S A. 2000; 97(19):10442-7.

11. Baiges A, Turon F, Simón-Talero M, Tasayco S, Bueno J, ZekriniK, et al. Congenital Extrahepatic Portosystemic Shunts (Abernethy Malformation): An International Observational Study. Hepatology. 2019; 71: 658-9.

12. Guérin F, Franchi Abella S, McLin V, Ackermann O, Girard M, Cervoni JP, Savale L, Hernandez-Gea V, Valla D, Hillaire S, Dutheil D, Bureau C, Gonzales E, Plessier A. Congenital portosystemic shunts: Vascular liver diseases: Position papers from the francophone network for vascular liver diseases, the French Association for the Study of the Liver (AFEF), and ERN-rare liver. Clinics and Research in Hepatology and Gastroenterology. 2020; Sep:44(4):452-459.

13. Franchi-Abella S, Branchereau S, Lambert V, Fabre M, Ste-imberg C, Losay J, et al. Complications of congenitalportosystemic shunts in children: therapeutic options and out-comes. J Pediatr Gastroenterol Nutr. 2010; 51:322-30. 
14. McLin V, Franchi-Abella S, Debray D, Korff S, Casotti V, Colledan M, et al. First report of the international registry of congenital porto-systemic shunts. J Pediatr Gastroenterol Nutr. 2019;68S1:678.

15. secuTrial ${ }^{\circledR}$ Web Site, a professional, GCP-compliant EDC system for collecting patient data in clinical research. https://www.secutrial.com/. Accessed 17 Sept 2021.

16. Documentation of KARNAK: a software gateway for medical image de-identification. https://osirix-foundation.github.io/karnak-documentation/. Accessed 24 Sept 2021.

17. Demonstration web site of KHEOPS platform for storage and distribution of medical images. http://www.kheops.online. Accessed 8 Sept 2021.

18. McLin V, Franchi-Abella S, Debray D, Korff S, Casotti V, et al. Congenital portosystemic shunts in children: preliminary results from the IRCPSS. 52nd Annual Meeting ESPGHAN (European Society for Pediatric Gastroenterology, Hepatology and Nutrition) (Glasgow, Scotland, du 05/06/2019 au 08/06/2019). http://hdl.handle.net/2078.1/216438

19. Franchi-Abella S, Gonzales E, Ackermann O, Branchereau S, Pariente D, Guérin F, on behalf of the International Registry of Congenital Portosystemic Shunt. Congenital portosystemic shunts: diagnosis and treatment. Abdominal Radiology 2018; Aug;43(8):2023-2036. 


\section{Figures}

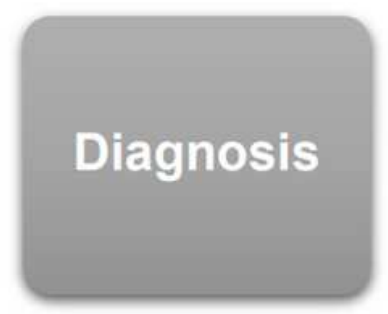

\section{Management \\ of CPSS and \\ associated \\ complications}

Management

and follow-up

after closure

\section{Figure 1}

Longitudinal follow-up of patients with CPSS

\section{Figure 2}

Data concepts, structure, and longitudinal collection of the complete data set UML presentation: package/class \& use case diagram - relationships: $0 . .1$ no instance or 1 instance, $0 .{ }^{*}$ zero or more instance, $1 .{ }^{*}$ at least one instance Purple: visits over time Yellow: clinical forms and imaging Green: specific forms at the Basic Data \& Diagnosis visit Red: specific forms at closure Grey: Specific forms at any time-point

\section{Figure 3}

secuTrial $\AA$ : overview of the data entry progress and completion that each available in real time for each center

secuTrial®: overview of subject visit plan and forms 